\title{
An epidemiological perspective on the future of direct-to-consumer personal genome testing
}

\author{
A Cecile JW Janssens ${ }^{*}$, Cornelia M van Duijn
}

\begin{abstract}
Personal genome testing is offered via the internet directly to consumers. Most tests that are currently offered use data from genome-wide scans to predict risks for multiple common diseases and traits. The utility of these tests is limited, predominantly because they lack predictive ability and clear benefits for disease prevention that are specific for genetic risk groups. In the near future, personal genome tests will likely be based on whole genome sequencing, but will these technological advances increase the utility of personal genome testing? Whole genome sequencing theoretically provides information about the risks of both monogenic and complex diseases, but the practical utility remains to be demonstrated. The utility of testing depends on the predictive ability of the test, the likelihood of actionable test results, and the options available for the reduction of risks. For monogenic diseases, the likelihood of known mutations will be extremely low in the general population and it will be a challenge to recognize new causal variants among all rare variants that are found using sequencing. For complex diseases, the predictive ability of genetic tests will be mainly restricted by the heritability of the disease, but also by the genetic complexity of the disease etiology, which determines the extent to which the heritability can be understood. Given that numerous genetic and non-genetic risk factors interact in the causation of complex diseases, the predictive ability of genetic models will likely remain modest. Personal genome testing will have minimal benefits for individual consumers unless major breakthroughs are made in the near future.
\end{abstract}

An increasing number of companies are offering healthrelated personal genome testing via the internet directly to consumers. Over time, these products have evolved from testing a few variants for a single health outcome to testing hundreds of thousands genetic variants genomewide for multiple outcomes simultaneously. These tests provide information about predisposition to drug response and risk predictions for a variety of diseases. For example, DeCODEme is currently predicting risks for 50 different diseases, traits and medication responses, Navigenics for 40 and 23 and Me for 66 . The outcomes predicted range from various cancers, to Alzheimer disease, to Warfarin response and eye color (accessed 14 July 2010). The utility of these tests is far from clear, not in the least because the predictive ability is still limited for most diseases, and risk predictions remain subject to change as long as new variants are being discovered [1-4].

To facilitate the discovery of new variants, nextgeneration whole genome sequencing is increasingly

\footnotetext{
* Correspondence: a.janssens@erasmusmc.nl
Department of Epidemiology, Erasmus University Medical Center Rotterdam,

* Correspondence: a.janssens@erasmusmc.nl
Department of Epidemiology, Erasmus University Medical Center Rotterdam, the Netherlands
}

(c) 2010 Janssens and van Duijn; licensee BioMed Central Ltd. This is an Open Access article distributed under the terms of the Creative

utilized in genetic research. The arrays used for genome wide scans include a very large but finite number of common variants covering the genome based on the principle of linkage disequilibrium. In contrast, whole genome sequencing documents the entire genome, base pair by base pair, and thus comprises more DNA variations, such as rare variants, copy number and structural variations with potentially larger effects on clinically relevant outcomes. Whole genome sequencing will be instrumental to discover more common variants implicated in complex outcomes, but may also reveal rare causal genetic variants for monogenic diseases that are private to specific populations or even to persons. Because whole genome sequencing gives a more complete coverage, it is beyond doubt that companies will consider the technique to predict predisposition to drug response and risks of complex and monogenic diseases. Whole genome sequencing is still rather expensive, particularly to obtain the high analytic validity that is required to make predictions at the individual level, but costs are decreasing rapidly. Yet, the question is whether these technological advances will also increase the utility 
of personal genome testing? This will depend on whether whole genome sequencing will be able to increase the predictive ability and the expected benefits of testing.

\section{Predictive ability of personal genome testing}

In the first place, the utility is determined by the question "will the personal genome testing have appreciable predictive ability to tell the future development of disease?" [5]. The predictive ability is evaluated for each disease separately and may differ considerably between diseases tested within the same scan, particularly between monogenic and complex traits. The difference in the predictive ability between monogenic and complex diseases is explained by two aspects of the genetic etiology, namely the heritability and the genetic complexity of the disease.

Monogenic diseases such as Huntington disease and cystic fibrosis are highly heritable. Although there may be multiple genetic and non-genetic factors influencing prognosis, mutations in a limited number of specific genes by themselves are sufficient causes of disease and testing the absence or presence of these mutations accurately predicts future disease development in families. Complex diseases on the other hand are caused by an intricate interplay of many genetic and non-genetic factors, such as smoking, alcohol consumption, diet and physical activity. The predictive ability of genetic risk models is determined by the combined effect of all genetic risk factors tested, and therefore indirectly by the frequency and effects of all variants included in the model. Empirical studies so far have shown that the predictive ability for most complex diseases is still moderate at best, which is for a large part explained by the relatively limited number of low-risk variants that have been discovered so far [6]. But even if all genetic variants were discovered in the future, still the predictive ability would be restricted by the fact that complex diseases are only partially heritable. Table 1 shows the wide range of heritability estimates that is observed for complex diseases and traits, ranging from $22 \%$ for happiness to $>99 \%$ for eye color. Figure 1 illustrates how the heritability relates to the maximum discriminative accuracy that can be obtained when all common and rare variants that constitute the heritability are identified $[7,8]$. This figure shows that theoretically an almost perfect genetic test will be possible for type 1 diabetes, but that for type 2 diabetes with a heritability of $26 \%$ and an estimated disease risk between $15-25 \%$ the discriminative accuracy will remain moderate.

Although the predictive ability of a genetic test for type 2 diabetes can become comparable to that of current non-genetic risk prediction models, this is only achieved when all genetic variants are identified.
Table 1 Heritability estimates of various complex diseases and traits

\begin{tabular}{llc}
\hline Disease or trait & Heritability & Reference \\
\hline Eye color & $>99 \%$ & {$[18]$} \\
Type 1 diabetes & $88 \%$ & {$[19]$} \\
Schizophrenia & $81 \%$ & {$[20]$} \\
Alzheimer's disease & $79 \%$ & {$[21]$} \\
Height & $70-87 \%(\mathrm{~m}), 68-85 \%(\mathrm{v})$ & {$[22]$} \\
Obesity & $65-84 \%(\mathrm{~m}), 64-79 \%(\mathrm{w})$ & {$[23]$} \\
Smoking persistence & $59 \%(\mathrm{~m}), 46 \%(\mathrm{w})$ & {$[24]$} \\
Anorexia nervosa & $56 \%$ & {$[25]$} \\
Rheumatoid arthritis & $53-65 \%$ & {$[26]$} \\
Panic disorder & $43 \%$ & {$[27]$} \\
Prostate cancer & $42 \%$ & {$[28]$} \\
Migraine & $40-50 \%$ & {$[29]$} \\
Heart attack & $38 \%(\mathrm{~m}), 57 \%(\mathrm{w})$ & {$[30]$} \\
Smoking initiation & $37 \%(\mathrm{~m}), 55 \%(\mathrm{w})$ & {$[24]$} \\
Depression & $37 \%$ & {$[31]$} \\
Colorectal cancer & $35 \%$ & {$[28]$} \\
Anxiety disorder & $32 \%$ & {$[27]$} \\
Homosexuality & $30 \%(\mathrm{~m}), 50-60 \%(\mathrm{w})$ & {$[32]$} \\
Breast cancer & $27 \%$ & {$[28]$} \\
Type 2 diabetes & $26 \%$ & {$[33]$} \\
Lung cancer & $26 \%$ & {$[28]$} \\
Happiness & $22 \%(\mathrm{~m}), 41 \%(\mathrm{w})$ & {$[34]$} \\
\hline
\end{tabular}

Heritability and frequency estimates are obtained from published studies and meta-analyses. $\mathrm{m}=$ men, $\mathrm{w}=$ women.

Underlying Figure 1 is the assumption that the total heritability can be explained. Whether this is a realistic assumption depends on the second major determinant: the complexity of the genetic etiology. It will be difficult to completely tease out genetic etiologies when there are many low-risk genetic variants implicated, such as the hundred that have been identified for blood cholesterol. These variants may interact with each other and with other non-genetic risk factors in many different ways. We still have little knowledge of the interactions that are anticipated from a biological perspective. So far, sophisticated genetic models that did consider interactions have not outperformed simple additive models $[9,10]$, but these studies have most likely lacked the power to investigate even two-factor interactions. Very complex interactions involving multiple genetic and non-genetic factors have been beyond the scope statistically and computationally, because increased or decreased risks due to complex interactions are difficult to detect. When ten variants are interacting, all cases and controls in epidemiological studies will have unique genetic profiles, making it impossible to accurately quantify risks of disease for specific combinations of variants [6]. And when more than 20 variants are interacting, even the world population may not be large enough to determine the risks for specific genetic profiles. Unless 


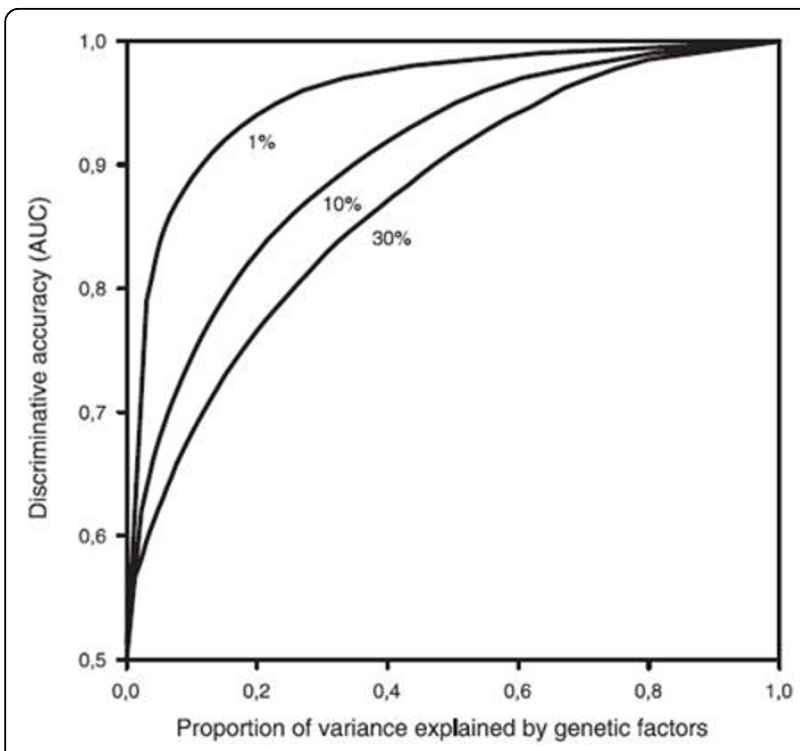

Figure 1 Relationship between the proportion of variance explained by genetic factors and the maximum discriminative accuracy of genetic testing. The discriminative accuracy, assessed as the Area Under the receiver operating characteristic Curve (AUC), is the extent to which predicted risks can discriminate between individuals who will develop the disease of interest and those who will not. The AUC is the probability that the test correctly identifies the person who will develop the disease from a pair of whom one will be affected and one will remain unaffected, and ranges from 0.5 (total lack of discrimination) to 1.0 (perfect discrimination). The numbers next to the smoothed lines refer to the risk of disease in the population. Reprinted with permission from ref 7 (Copyright 2006, Wolters Kluwer Health).

the assumption of additive effects of genetic and nongenetic risk factors is correct, these simple examples show that the more complex the genetic etiology of a trait, the less likely it is that the genetic contribution will be fully understood in all its complexity.

The impact of heritability and genetic complexity on the potential predictive ability of diseases is outlined in Figure 2. When diseases are highly heritable and have a simple genetic etiology, e.g., as in monogenetic disorders, genetic testing will be accurate and very predictive. Whether these variants are mutations, haplotypes, copy number variations, insertion/deletions, or even missing or extra copies of an entire chromosome, the presence of the variants means that one will develop the disease and the absence that one will not. Also traits that are predominantly determined by one or a few variants, such as human eye color, may be predicted with relatively high accuracy [11]. When diseases are only partially influenced by genetic factors and the heritability is in the lower ranges, the predictive ability of tests that consider genetic variants only will never be very accurate when nongenetic risk factors are not included in the test. There are however examples of traits with low heritability and

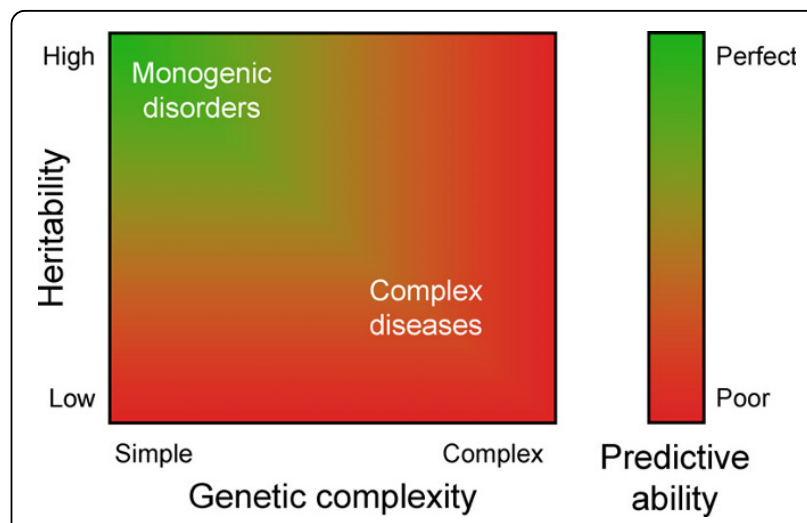

Figure 2 Relationship between the heritability, genetic complexity and predictive ability of personal genome testing.

simple genetic etiology for which genetic testing may be clinically relevant. These include genetic effects on responses to medication, where one or a few variants, e.g., in the cytochrome P450 genes, have a large effect but do by themselves not fully determine treatment response. In contrast, when diseases are highly heritable but genetically complex, the predictive ability may not come close to the theoretical maximum, because the complexity hampers accurate assessment of the risks associated with the specific combinations of variants.

\section{Expected benefits of personal genome testing}

The utility of personal genome testing is also determined by the expected benefits for the individual who undergoes testing. The range of potential benefits for genome wide tests is broad and includes not only enabling decisions about preventive or therapeutic interventions, but also more subjective benefits such as enhancing motivation, making plans for the future, and simply knowing one's risks of disease. These subjective benefits of testing have led to the recognition that in addition to clinical and public health utility, there is personal utility that will likely vary among consumers [12-14].

A challenge in the evaluation of the expected benefits is the fact that genome-wide scans, and in the future also whole genome sequencing, address multiple diseases at the same time and typically are not advertised and ordered for the prediction of one specific disease. This means that the benefits of testing should be evaluated across all conditions and traits that are tested, because it is not known in advance for which disease one will benefit from testing. This feature increases the utility of personal genome testing, as by definition, the probability of carrying a mutation or being at high risk for at least one disease is higher than that for one specific disorder.

However, the expected benefits of testing not only depend on what benefits may occur, but also on the 
likelihood of their occurrences. Even though the benefits of testing may be higher when more diseases are tested for, the likelihood of having at least some benefit may still be quantitatively low. For example, 23andme tests three mutations in BRCA $1 / 2$ of the thousands that have been documented, but which are found solely among persons from Ashkenazi Jewish descent. The probability that women with no Ashkenazi Jewish roots will learn that they have one of these mutations is virtually zero. Similarly, many mutations for other monogenic traits are specific to populations or families, implying that also individuals with a family history of disease may test negative for documented mutations. Whole genome sequencing will likely identify new mutations in these individuals, but their impact on disease risk is unknown without evaluation in other affected and non-affected relatives. Thus, testing for rare variants with strong effects has little relevance in the absence of family specific information. Predisposition to drug responses is another example where a priori the expected benefits of testing are low, because the probability of these benefits is conditional on developing an indication for the specific drug and the deleterious gene variants may be population specific.

But also for complex diseases, the likelihood of benefits may be disappointingly low. The presumed rationale for learning about one's risk is that elevated risks can motivate to adopt a healthier lifestyle or other preventive strategies, but genetic risk models with moderate predictive ability are not able to distinguish sizeable subgroups with substantially elevated risks. For example, in a large-population-based cohort the average risk of type 2 diabetes was $20 \%$ and the observed range of risks predicted by a model that included 18 low-risk susceptibility variants was 7 to $45 \%$ [15]. If this test were offered by companies to consumers, approximately $5 \%$ of the consumers would learn that their risks are higher than $30 \%$ but nobody that their risk are higher than $50 \%$. Hence, consumers who would only be motivated when their genetic risks were at least $50 \%$ could have known a priori that this motivational benefit should not be expected from this test. To learn whether personal genome testing might motivate, consumers should first ask themselves what risk increase would make them change their behavior and then see whether the test has sufficient predictive ability to tell that risk. Because it is argued that the key to the success of DTC companies is clarity and transparency [14,16], consumers should have insight in the expected benefits and the predictive ability prior to purchasing.

\section{Concluding remarks}

Even though most direct-to-consumer companies are still offering personal genome testing on the basis of genome-wide scans, it is clear that this technique will be outdated as soon as whole genome sequencing becomes affordable to consumers. Standard genome-wide scans will increasingly fail to cover the latest variants for the prediction of complex diseases when whole genome sequencing is used for variant discovery, and they do not cover variants for monogenic traits. Whole genome sequencing covers the entire DNA sequence, which means that all DNA variations can be read including common and rare variants implicated in monogenic and complex outcomes.

For monogenic diseases, whole genome sequencing will have the same predictive ability as tests of known mutations that are currently conducted in the clinical genetics practice. Whole genome sequencing can simultaneously test for many monogenic diseases, which increases the likelihood that individuals might benefit from knowing their DNA for at least one disease. This feature of whole genome sequencing certainly contributes to a strong sense of personal utility. However, in the absence of a positive family history or early symptoms, the a priori probability of carrying a known mutation will be low. At the same time, whole genome sequencing will also reveal numerous mutations with unknown impact that have never been seen before. These unknown mutations are difficult to interpret. High-throughput algorithms that combine information from biology, bioinformatics, proteomics and population genetics might be developed in the future to predict the effect of the many variants encountered, but at present one needs family, epidemiological or functional studies to quantify the deleterious effect of such mutations.

For complex diseases, whole genome sequencing will also certainly outperform prediction based on genomewide scans, because more variants are captured. Yet, because complex diseases are only partly determined by genetic variants or are genetically too complex, it should not be expected that prediction of complex diseases becomes markedly better. When the predictive ability remains limited and prevention is effective even in the absence of a genetically increased risk, the likelihood that test results will lead to any benefit or intelligible decisions at the a personal level, is very low.

Does this mean that whole genome sequencing will have no utility at all? This is clearly not the case. There are many tests in health care that have moderate predictive ability for the individual, but these are found useful to make decisions about prevention or treatment for larger groups of people. Similarly, there will be numerous opportunities in health care in which whole genome sequencing likely may be implemented to improve health outcomes in patients and populations; opportunities where a moderate predictive ability is sufficient and the expected benefits are higher. Moderate predictive ability may be sufficient in population-based screening programs, such as breast cancer mammography 
screening, where whole genome sequencing could be used to differentiate in starting age or frequency of monitoring [17]. Expected benefits may be larger when tests are targeted to specific at-risk populations, e.g., to identify the genetic cause of congenital disorders in newborns, to search and subsequently test private mutations in families with a positive history of hereditary disease, or to test predisposition to specific drug responses upon indication of symptoms.

Undoubtedly, whole genome sequencing will be implemented in health care when it leads to better health outcomes for populations, even if at the personal level the benefits are modest and many individuals receive a wrong or unnecessary preventive or therapeutic intervention. Health care payers will finance predictive tests that benefit populations at large, but it is unlikely that individual consumers will massively spend money on expensive tests that have moderate predictive ability and unclear personal benefits.

Received: 13 August 2010 Accepted: 4 October 2010

Published: 4 October 2010

\section{References}

1. Mihaescu R, van Hoek M, Sijbrands EJ, Uitterlinden AG, Witteman JC, Hofman A, van Duijn CM, Janssens AC: Evaluation of risk prediction updates from commercial genome-wide scans. Genet Med 2009, 11:588-94.

2. Patch C, Sequeiros J, Cornel MC: Direct to consumer genetic tests. Eur J Hum Genet 2009, 17:1111.

3. Hunter DJ, Khoury MJ, Drazen JM: Letting the genome out of the bottlewill we get our wish? N Engl J Med 2008, 358:105-7.

4. van Ommen GB, Cornel MC: Recreational genomics? Dreams and fears on genetic susceptibility screening. Eur J Hum Genet 2008, 16:403-4.

5. Hamburg MA, Collins FS: The Path to Personalized Medicine. N Engl J Med 2010, 363:301-4.

6. Janssens ACJW, Van Duijn CM: Genome-based prediction of common diseases: advances and prospects. Hum Mol Genet 2008, 17:R166-R173.

7. Janssens ACJW, Aulchenko YS, Elefante S, Borsboom GJJM, Steyerberg EW, Van Duijn CM: Predictive testing for complex diseases using multiple genes: fact or fiction? Genet Med 2006, 8:395-400.

8. Wray NR, Yang J, Goddard ME, Visscher PM: The genetic interpretation of area under the ROC curve in genomic profiling. PLoS Genet 2010, 6: e1000864.

9. Liu F, van Duijn K, Vingerling JR, Hofman A, Uitterlinden AG, Janssens AC, Kayser M: Eye color and the prediction of complex phenotypes from genotypes. Curr Biol 2009, 19:R192-R193.

10. Yu W, Liu T, Valdez R, Gwinn M, Khoury MJ: Application of support vector machine modeling for prediction of common diseases: the case of diabetes and pre-diabetes. BMC Med Inform Decis Mak 2010, 10:16.

11. Liu F, Ikram MA, Janssens AC, Schuur M, de Koning I, Isaacs A, Struchalin M, Uitterlinden AG, den Dunnen JT, Sleegers K, Bettens K, Van Broeckhoven C, van Swieten J, Hofman A, Oostra BA, Aulchenko YS, Breteler MM, van Duijn CM: A Study of the SORL1 Gene in Alzheimer's Disease and Cognitive Function. J Alzheimers Dis 2009, 18:51-64.

12. Grosse SD, McBride CM, Evans JP, Khoury MJ: Personal utility and genomic information: look before you leap. Genet Med 2009, 11:575-6.

13. Foster MW, Mulvihill JJ, Sharp RR: Evaluating the utility of personal genomic information. Genet Med 2009, 11:570-4.

14. Helgason A, Stefansson K: The past, present, and future of direct-toconsumer genetic tests. Dialogues Clin Neurosci 2010, 12:61-8.

15. van Hoek M, Dehgan A, Witteman JCM, Van Duijn CM, Uitterlinden AG, Oostra BA, Hofman A, Sijbrands EJ, Janssens AC: Predicting type 2 diabetes based on polymorphisms from genome wide association studies: a population-based study. Diabetes 2008, 57:3122-8.
16. Khoury MJ, McBride CM, Schully SD, loannidis JP, Feero WG, Janssens AC, et al: The Scientific Foundation for personal genomics: recommendations from a National Institutes of Health-Centers for Disease Control and Prevention multidisciplinary workshop. Genet Med 2009, 11:559-67.

17. Pharoah PD, Antoniou AC, Easton DF, Ponder BA: Polygenes. Polygenes, risk prediction, and targeted prevention of breast cancer. $N$ Engl J Med 2008, 358:2796-803.

18. Zhu G, Evans DM, Duffy DL, Montgomery GW, Medland SE, Gillespie NA, Ewen KR, Jewell M, Liew YW, Hayward NK, Sturm RA, Trent JM, Martin NG: A genome scan for eye color in $\mathbf{5 0 2}$ twin families: most variation is due to a QTL on chromosome 15q. Twin Res 2004, 7:197-210.

19. Hyttinen V, Kaprio J, Kinnunen L, Koskenvuo M, Tuomilehto J: Genetic liability of type 1 diabetes and the onset age among 22,650 young Finnish twin pairs: a nationwide follow-up study. Diabetes 2003 , 52:1052-5.

20. Sullivan PF, Kendler KS, Neale MC: Schizophrenia as a complex trait: evidence from a meta-analysis of twin studies. Arch Gen Psychiatry 2003, 60:1187-92.

21. Gatz M, Reynolds CA, Fratiglioni L, Johansson B, Mortimer JA, Berg S, Fiske A, Pedersen NL: Role of genes and environments for explaining Alzheimer disease. Arch Gen Psychiatry 2006, 63:168-74.

22. Silventoinen K, Sammalisto S, Perola M, Boomsma DI, Cornes BK, Davis C, Dunkel L, De Lange M, Harris JR, Hjelmborg JV, Luciano M, Martin NG, Mortensen J, Nisticò L, Pedersen NL, Skytthe A, Spector TD, Stazi MA, Willemsen G, Kaprio J: Heritability of adult body height: a comparative study of twin cohorts in eight countries. Twin Res 2003, 6:399-408.

23. Schousboe K, Willemsen G, Kyvik KO, Mortensen J, Boomsma DI, Cornes BK, Davis CJ, Fagnani C, Hjelmborg J, Kaprio J, De Lange M, Luciano M, Martin NG, Pedersen N, Pietiläinen KH, Rissanen A, Saarni S, Sørensen TI, Van Baal GC, Harris JR: Sex differences in heritability of BMI: a comparative study of results from twin studies in eight countries. Twin Res 2003, 6:409-21

24. Li MD, Cheng R, Ma JZ, Swan GE: A meta-analysis of estimated genetic and environmental effects on smoking behavior in male and female adult twins. Addiction 2003, 98:23-31.

25. Bulik CM, Sullivan PF, Tozzi F, Furberg H, Lichtenstein P, Pedersen NL: Prevalence, heritability, and prospective risk factors for anorexia nervosa. Arch Gen Psychiatry 2006, 63:305-12.

26. MacGregor AJ, Snieder H, Rigby AS, Koskenvuo M, Kaprio J, Aho K, Silman AJ: Characterizing the quantitative genetic contribution to rheumatoid arthritis using data from twins. Arthritis Rheum 2000, 43:30-7.

27. Hettema JM, Neale MC, Kendler KS: A review and meta-analysis of the genetic epidemiology of anxiety disorders. Am J Psychiatry 2001, 158:1568-78.

28. Lichtenstein P, Holm NV, Verkasalo PK, Iliadou A, Kaprio J, Koskenvuo M, Pukkala E, Skytthe A, Hemminki K: Environmental and heritable factors in the causation of cancer-analyses of cohorts of twins from Sweden, Denmark, and Finland. N Engl J Med 2000, 343:78-85.

29. Ligthart L, Boomsma DI, Martin NG, Stubbe JH, Nyholt DR: Migraine with aura and migraine without aura are not distinct entities: further evidence from a large Dutch population study. Twin Res Hum Genet 2006, 9:54-63.

30. Zdravkovic S, Wienke A, Pedersen NL, Marenberg ME, Yashin Al, De Faire U: Heritability of death from coronary heart disease: a 36-year follow-up of 20966 Swedish twins. J Intern Med 2002, 252:247-54.

31. Sullivan PF, Neale MC, Kendler KS: Genetic epidemiology of major depression: review and meta-analysis. Am J Psychiatry 2000, 157:1552-62.

32. Kirk KM, Bailey JM, Dunne MP, Martin NG: Measurement models for sexual orientation in a community twin sample. Behav Genet 2000, 30:345-56.

33. Poulsen $\mathrm{P}$, Kyvik KO, Vaag A, Beck-Nielsen H: Heritability of type II (noninsulin-dependent) diabetes mellitus and abnormal glucose tolerance-a population-based twin study. Diabetologia 1999, 42:139-45.

34. Bartels M, Saviouk V, de Moor MH, Willemsen G, van Beijsterveldt TC, Hottenga JJ, de Geus EJ, Boomsma DI: Heritability and genome-wide linkage scan of subjective happiness. Twin Res Hum Genet 2010, 13:135-42.

doi:10.1186/2041-2223-1-10

Cite this article as: Janssens and van Duijn: An epidemiological perspective on the future of direct-to-consumer personal genome testing. Investigative Genetics 2010 1:10. 